\title{
QUADRATIC JORDAN ALGEBRAS WHOSE ELEMENTS ARE ALL INVERTIBLE OR NILPOTENT
}

\author{
KEVIN MCCRIMMON
}

\begin{abstract}
We prove that if $\mathfrak{J}$ is a unital quadratic Jordan algebra whose elements are all either invertible or nilpotent, then modulo the nil radical $\mathfrak{N}$ the algebra $\mathfrak{I} / \mathfrak{N}$ is either a division algebra or the Jordan algebra determined by a traceless quadratic form in characteristic 2 . We also show that if $\mathfrak{A}$ is an associative algebra with involution whose symmetric elements are either invertible or nilpotent, then modulo its radical $\mathfrak{R} / \mathfrak{R}$ is a division algebra, a direct sum of anti-isomorphic division algebras, or a split quaternion algebra.
\end{abstract}

In [1] N. Jacobson filled a gap in A. A. Albert's structure theory for finite-dimensional Jordan algebras over a field $\Phi$ of characteristic $\neq 2$ by showing that if $\mathfrak{J}$ was almost nil (in the sense that every element could be written as $\alpha 1+z$ for $\alpha \in \Phi$ and $z$ nilpotent) then the set $\mathfrak{N}$ of nilpotent elements formed an ideal (see also [2, p. 198]). This was extended by J. M. Osborn [9] to the case of an arbitrary Jordan algebra without 2-torsion in which every element was invertible or nilpotent. Such algebras occur naturally in the structure theory of Jordan algebras: D. L. Morgan has shown [8, Theorem 2.1] that if $e$ is a primitive idempotent in a Jordan algebra $\mathfrak{J}$ with d.c.c. on inner ideals, then the Peirce subalgebra $\mathfrak{I}_{1}(e)$ has this property.

The result fails in characteristic 2-the Jordan algebras $J(Q, 1)$ of traceless quadratic forms $Q$ satisfy $x^{2}=Q(x) 1$ for all $x$, so either $Q(x)=0$ (and $x$ is nilpotent) or $Q(x) \neq 0$ (and $x$ is invertible). A particular case: $\Im$ consists of all matrices $\left(\begin{array}{cc}\alpha & \beta \\ \gamma & \alpha\end{array}\right)$, so $x^{2}=(\operatorname{det} x) 1$ for $\operatorname{det} x=\alpha^{2}+\beta \gamma$. However, in [4] it was shown that these were essentially the only counterexamples: any finite-dimensional almost nil quadratic Jordan algebra over a field $\Phi$ with more than two elements has nil radical $\mathfrak{N}$ such that $\mathfrak{I} / \mathfrak{N}$ is either $\Phi 1$ or $J(Q, 1)$ for a nondegenerate traceless $Q$. (We refer to [3] for all basic facts about quadratic Jordan algebras over an arbitrary ring of scalars $\Phi$.)

Received by the editors January 28, 1972.

AMS 1970 subject classifications. Primary 17C10, 17C50; Secondary 16A28, 16A68.

Key nords and phrases. Quadratic Jordan algebra, invertible element, nilpotent element.

c. American Mathematical Society 1972 
In the present paper we will extend this result to arbitrary quadratic Jordan algebras whose elements are either invertible or nilpotent, thus establishing Osborn's result for quadratic Jordan algebras.

THEOREM 1. The quadratic Jordan algebras in which every element is either invertible or nilpotent are precisely those for which $\mathfrak{I} / \mathfrak{N}(\mathfrak{N}$ the nil radical) is a division algebra or an algebra $J(Q, 1)$ determined by a traceless nondegenerate quadratic form $Q$ over a field of characteristic 2.

Since an element $x$ of $\mathfrak{J}$ is nilpotent or invertible if and only if its image $\bar{x}$ in $\mathfrak{J}=\mathfrak{J} / \mathfrak{N}$ is nilpotent or invertible, when $\mathfrak{J} / \mathfrak{N}$ has the stated form, the elements of $\mathfrak{J}$ are invertible or nilpotnet. We concentrate on showing the converse. If noninvertible elements are nilpotent the Jacobson radical $\operatorname{Rad} \mathfrak{I}$ coincides with the nil radical $N(\mathfrak{I})$ (always $N(\mathfrak{J}) \subset \operatorname{Rad} \mathfrak{J}$, and $\operatorname{Rad} \mathfrak{I}$ never contains invertible elements). Further, $\mathfrak{J}$ shares the same property as $\mathfrak{J}$ but in addition is semisimple, so it suffices to prove

THEOREM $1^{\prime}$. If $\mathfrak{J}$ is a semisimple quadratic Jordan algebra in which every element is invertible or nilpotent, then $\mathfrak{J}$ is either a division algebra or an algebra $J(Q, 1)$ determined by a traceless nondegenerate quadratic form over a field of characteristic 2.

Proof. If all nonzero elements are invertible, $\mathfrak{J}$ is a division algebra. Therefore we will always assume the set $Z$ of nilpotent elements is not zero. Our goal is to find an isotope $\tilde{\mathfrak{J}}$ which has capacity two and use Osborn's Capacity Two Theorem to get $\widetilde{\mathfrak{J}} \cong J(Q, 1)$.

Our first observation is that all $z$ in $Z$ square to zero, because in general

LEMMA 1. If $U_{z} a$ is nilpotent for each $a \in \mathfrak{J}$ then $z^{2} \in \operatorname{Rad} \mathfrak{J}$.

Proof. Since the radical Rad $\mathfrak{J}$ consists of the elements quasi-invertible in all homotopes $\mathfrak{J}^{(a)}[6]$, it suffices if $z^{2}$ is nilpotent in each $\mathfrak{J}^{(a)}$. Recall that an element is nilpotent if and only if its $U$-operator is nilpotent; but the $U$-operator of $z^{2}$ in $\mathfrak{J}^{(a)}$ is $U_{z^{2}}^{(a)}=U_{z^{2}} U_{a}=U_{z} U_{z} U_{a}$, which is nilpotent since $S T$ is nilpotent iff $T S$ is nilpotent, and $\left(U_{z} U_{a}\right) U_{z}=U_{U(z) a}$ is nilpotent by hypothesis.

$Z$ cannot be an ideal if $\mathfrak{J}$ is semisimple, yet for any $z \in Z$ and $x \in \mathfrak{J}$, both $U_{x} z$ and $U_{z} x$ belong to $Z$ (they cannot be invertible, since $U_{x} y$ is invertible iff $x$ and $y$ are, so they must be nilpotent), so the only way $Z$ can fail to be an ideal is by not being closed under addition: $Z+Z \notin Z$. This means some sum of nilpotent elements $z, w$ adds up to an invertible element $z+w=u$.

Already at this stage we can conclude $\mathfrak{I}$ has characteristic $2(2 \mathfrak{I}=0)$, and thus recapture Osborn's result. Indeed, if $z+w=u$ for $z^{2}=w^{2}=0$ then $1+w$ is invertible but $U_{1+u^{2}}$ is nilpotent, so $0=U_{1+w}^{-1}\left\{U_{1+w} z\right\}^{2}=$ $U_{1-u}^{-1} U_{1+i} U_{z}(1+w)^{2}=U_{z}(1+2 w)=z^{2}+2 U_{z} w=2 U_{z} w=2 U_{u} w \quad$ as $U_{w} w=$ $w^{3}=0, U_{z, w^{\prime}} w^{\prime}=z \cdot w^{2}=0$ ) and therefore $2 w=0$ for $w \neq 0$. Since the centroid 
of our $\mathfrak{I}$ must be a field, this gives $2=0$. Instead of assuming characteristic 2 , we prefer to keep our calculations general (the reader will see how they can be simplified if $2 \mathfrak{I}=0$ ).

Once the elements of $Z$ all square to zero, we can show that any $z$ with $z+w=u$ invertible is von Neumann-regular and spawns an idempotent.

LEMMA 2. In any quadratic Jordan algebra, if $z+w=u$ is invertible for $z^{2}=z^{3}=w^{2}=0$ then $z=U_{z} y, y=U_{y} z$ for $y=U_{u^{-1}} z$. Whenever $z=U_{z} y$ for $z^{2}=y^{2}=0$ then $e=z \circ y$ is an idempotent with $U_{e} z=z$, hence $e \neq 0$ if $z \neq 0$.

Proof. For the first assertion, $1=U_{u}^{-1} u^{2}=U_{u}^{-1}(z \circ w)=U_{u}^{-1}(z \circ u)$ (as $\left.z \circ z=2 z^{2}=0\right)=u^{-1} \circ z$, so

$$
\begin{aligned}
z & =U_{1} z=U_{u^{-1}{ }_{\circ} z} z=\left\{U_{u^{-1}} U_{z}+U_{z} U_{u^{-1}}+U_{u^{-1}, z}^{2}-U_{u^{-2}, z^{2}}\right\} z \\
& =U_{z} U_{u^{-1}}
\end{aligned}
$$

since $U_{z} z=z^{3}=0,\left\{u^{-1} z \quad z\right\}=u^{-1} \circ z^{2}=0, z^{2}=0$. Further,

$$
U_{y} z=\left(U_{u^{-1}} U_{z} U_{u^{-1}}\right) z=U_{u^{-1}} z=y .
$$

For the second assertion, $e^{2}=(z \circ y)^{2}=U_{z} y^{2}+U_{y} z^{2}+y \circ U_{z} y=0+0+$ $y \circ z=e$ is idempotent with

$$
U_{e} z=U_{z \circ y} z=\left\{U_{z} U_{y}+U_{y} U_{z}+U_{z, y}^{2}-U_{y^{2}, z^{2}}\right\} z=U_{z} U_{y} z
$$

(here $\left.z^{3}=U_{z^{2}}=U_{z} U_{z} y=U_{z^{2}} y=0,\{y z z\}=y \circ z^{2}=0, z^{2}=0\right)=U_{z} U_{y}\left(U_{z} y\right)=$ $U_{U(z) y} y=U_{z} y=z$.

In our situation the only idempotent other than zero is 1 , so from $z+w=u$ we have found

$$
z=U_{z} y, \quad y=U_{y} z, \quad z^{3}=z^{2}=y^{2}=0, \quad z \circ y=1 .
$$

The isotope we are looking for is $\tilde{\mathfrak{I}}=\mathfrak{I}^{(n)}, v=1+y+2 z$. The element $v=1+w(w=y+2 z)$ is invertible with inverse $v^{-1}=-1+w(=-1+y+2 z)$ since $w^{2}=2 y \circ z=2$. Here $e=z$ is an idempotent in $\tilde{\mathfrak{J}}$, since $e^{\tilde{2}}=U_{e} v=$ $U_{z}(1+y+2 z)=z^{2}+U_{z} y+2 z^{3}=0+z+0=e$. The element $c=1-2 z$ belongs to the Peirce space $\tilde{\mathfrak{J}}_{1 / 2}(e)$ since $e \tilde{\circ} c=\left\{\begin{array}{lll}z & v 1-2 z\}=z \circ v-4 U_{z} v=\{2 z+\end{array}\right.$ $\left.z \circ y+4 z^{2}\right\}-4 z=1-2 z=c$. Further, $c$ squares to $\tilde{1}$ since $c^{\tilde{2}}=U_{c} v=$ $U_{c}\left(c^{-1}+y\right)\left(1-2 z\right.$ and $1+2 z$ are inverses if $\left.z^{2}=0\right)=c+U_{1-2 z} y=(1-2 z)+$ $\left(y-2 z \circ y+4 U_{z} y\right)=1-2 z+y-2+4 z=-1+y+2 z=v^{-1}=1^{(v)}=\tilde{1}$. The condition that noninvertible elements in $\mathfrak{J}=\left\{\mathfrak{J}^{(v)}\right\}^{\left(v^{-2}\right)}=\tilde{\mathfrak{J}}_{\tilde{J}}^{\left(v^{-2}\right)}$ square to zero becomes the condition that noninvertible elements in $\tilde{\mathfrak{J}}$ kill $c$,

$x$ not invertible in $\tilde{\mathfrak{J}} \Rightarrow \tilde{U}_{x} c=0$

since $0=x^{2}=\widetilde{U}_{x} v^{-2}$ where $v^{-2}=(w-1)^{2}=w^{2}-2 w+1=3-2 w=1-2 v^{-1}=$ $(1-2 z)+2 z-2 v^{-1}=c+2 e-2 \tilde{1}=c-2 f$ gives $\tilde{U}_{x}(c-2 f)=0$; in particular, $\tilde{U}_{f}(c-2 f)=-2 f=0$, so that $\tilde{U}_{x} c=0$ for such $x$. 
To apply the Capacity Two Theorem we need to know that $\tilde{\mathfrak{I}}_{1}$ and $\tilde{\mathfrak{I}}_{0}$ are division algebras and $\tilde{\mathfrak{J}}$ contains no trivial elements. But $\tilde{\mathfrak{J}}$ is semisimple since $\mathfrak{J}$ is, and a semisimple algebra contains no trivial elements [5], so we can apply

LEMMA 3. Let $\mathfrak{J}$ be a quadratic Jordan algebra without trivial elements, $e$ an idempotent, and $c \in \mathfrak{J}_{1 / 2}(e)$ an element with $c^{2}=1$ such that

$$
x \text { not invertible in } \mathfrak{J} \text { implies } U_{x} c=0 \text {. }
$$

Then $\mathfrak{J}$ is semisimple of connected capacity 2 , and the involution $U_{c}$ is trivial on $\mathfrak{J}_{1 / 2}(e)$.

Proof. Here $U_{c}$ is an automorphism of $\mathfrak{J}$ of period 2 taking $e$ into $f=$ $1-e$; clearly $c$ strongly connects $e$ and $f$, and $\mathfrak{I}_{0}=U_{c} \mathfrak{J}_{1}$ will be a division algebra if $\mathfrak{J}_{1}$ is. All that remains is to show $\mathfrak{J}_{1}$ is a division algebra and $U_{c}$ is trivial on $\mathfrak{I}_{1 / 2}$.

Suppose $a$ is not invertible in $\mathfrak{J}_{1}$. Then $a, f$, and $a+f$ are not invertible in $\mathfrak{J}$, so by hypothesis $0=U_{a+f} c-U_{a} c-U_{f} c=\{a c f\}=a \circ(c \circ f)=a \circ c$. But if $a \circ c=0$ then $a$ is trivial: $U_{a} \mathfrak{J}=U_{a} \mathfrak{J}_{1}=U_{a}\left(U_{c} \mathfrak{J}_{0}\right)=U_{a} \circ \mathfrak{J}_{0}=0$. By hypothesis the only trivial element $\mathfrak{J}$ can have is zero, so the only element of $\mathfrak{I}_{1}$ which is not invertible is zero, showing $\mathfrak{J}_{1}$ to be a division algebra.

To show the involution $U_{c}$ is the identity on $\mathfrak{J}_{1 / 2}$, take any element $x$ in $\mathfrak{J}_{1 / 2}$ and consider $y=a_{1}+x+f\left(\right.$ where $x^{2}=a_{1}+a_{0}$ for $a_{i}$ in $\left.\mathfrak{I}_{i}\right)$. This modification of $x$ is noninvertible since

$$
\begin{aligned}
U_{y}\left(e-x+a_{0}\right)= & \left\{U_{a_{1}}+U_{x}+U_{f}+U_{a_{1}, x}+U_{a_{1}, f}+U_{x, f}\right\}\left(e-x+a_{0}\right) \\
= & a_{1}^{2}+\left(U_{x} e-x^{3}+U_{x} a_{0}\right)+a_{0}+\left(a_{1} \circ x-a_{1} \circ x^{2}\right) \\
& -a_{1} \circ x+\left(-x^{2} \circ f+x \circ a_{0}\right) \quad \text { (by the Peirce relations) } \\
= & a_{1}^{2}+a_{0}-x^{3}+a_{1}^{2}+a_{0}+x^{3}-2 a_{1}^{2}-x^{3}-2 a_{0}+x^{3} \\
= & \left.0 \quad \text { (using the fact that } U_{x} a_{i}=a_{j}^{2}, a_{i} \circ x=x^{3}\right) .
\end{aligned}
$$

Therefore by the assumed property of $\mathfrak{J}$ we have

or

$$
U_{y} c=0 \text {, }
$$

$$
\begin{aligned}
0 & =U_{f} U_{y} c=U_{f} U_{y} V_{f} c=\left\{-V_{f} U_{y} U_{f}+U_{U(f) y, V(f) y}\right\} c \\
& =-0+U_{f, x+2 f} c=U_{f, x} c=U_{f}(x \circ c),
\end{aligned}
$$

the component of $x \circ c$ in $\mathfrak{J}_{0}$. Thus $U_{c} x=c \circ U_{f}(c \circ x)-x \circ U_{e}\left(c^{2}\right)=$ $-x \circ e=-x$. But since $U_{c} c=c^{3}=c$ we have $c=-c, 1=-1$, so also $U_{c} x=x$.

Once $\tilde{\mathfrak{J}}$ is strongly connected semisimple of capacity two with trivial involution $\tilde{U}_{c}=I$ on $\widetilde{\mathfrak{J}}_{1 / 2}$, we can apply Osborn's Capacity Two Theorem [3] to conclude $\tilde{\mathfrak{J}} \cong J(\tilde{Q}, \tilde{1})$ for a nondegenerate quadratic form $\tilde{Q}$. Thus 
$\mathfrak{I} \cong \mathfrak{I}(\widetilde{Q}, \tilde{1})^{\left(v^{-2}\right)}=J(Q, 1)$ is also determined by a nondegenerate quadratic form. The condition that each element be invertible $(Q(x) \neq 0)$ or nilpotent $(Q(x)=T(x)=0)$ then implies $Q$ is traceless of characteristic 2 .

REMARK. Rather than prove that the involution is trivial, one can consider the other possibility in Osborn's Theorem: $\mathfrak{I} \cong \mathfrak{H}\left(\Delta_{2}, \Delta_{0}\right)$ with $e \cong\left(\begin{array}{ll}1 & 0 \\ 0 & 0\end{array}\right), c \cong\left(\begin{array}{ll}0 & 1 \\ 1 & 0\end{array}\right)$, for $\Delta$ an associative division algebra with nontrivial involution and ample subspace $\Delta_{0}$. This possibility can be ruled out as follows: any $y=\left(\begin{array}{cc}\delta \bar{\delta} & \delta \\ \bar{\delta} & 1\end{array}\right)$ is noninvertible (it kills $\left.\left(\begin{array}{cc}1 & \overline{-\delta} \\ \bar{\delta} \delta\end{array}\right)\right)$, so by hypothesis

$$
0=y c y=\left(\begin{array}{cc}
\delta \bar{\delta} & \delta \\
\bar{\delta} & 1
\end{array}\right)\left(\begin{array}{ll}
0 & 1 \\
1 & 0
\end{array}\right)\left(\begin{array}{cc}
\delta \bar{\delta} & \delta \\
\bar{\delta} & 1
\end{array}\right)=\left(\begin{array}{cc}
\delta(\delta+\bar{\delta}) \bar{\delta} & \delta(\delta+\bar{\delta}) \\
(\delta+\bar{\delta}) \bar{\delta} & \delta+\bar{\delta}
\end{array}\right) .
$$

Thus $\delta+\bar{\delta}=0$ for all $\delta$, so $1=-1, \bar{\delta}=\delta$ contradicts the nontriviality of the involution on $\Delta$.

Osborn applied this result to $\mathfrak{J}=\mathfrak{H}(\mathfrak{H}, *)$ to show that if the symmetric elements of an associative algebra $\mathfrak{H}$ in characteristic $\neq 2$ are all invertible or nilpotent, then $\mathfrak{A}$ is a division algebra, direct sum of antiisomorphic division algebras, or a split quaternion algebra. We will obtain the same result for arbitrary characteristics.

Recall that a subspace $\mathfrak{J}$ of symmetric elements of an algebra $\mathfrak{A}$ is ample if it contains 1 and $x \mathfrak{I} x * \subset \mathfrak{J}$ for all $x$ in $\mathfrak{A}$ (therefore it contains all norms $x x^{*}$ and all traces $x+x^{*}$ ). If $\frac{1}{2} \in \Phi$ then the only ample subspace of $\mathfrak{H}(\mathfrak{A}, *)$ is $\mathfrak{H}(\mathfrak{A}, *)$ itself. An ample subspace is automatically a Jordan subalgebra.

THEOREM 2. If $\mathfrak{A}$ is a unital associative algebra with involution and $\mathfrak{J}$ an ample subspace of $\mathfrak{H}(\mathfrak{A}, *)$ such that each element of $\mathfrak{J}$ is invertible or nilpotent, then $\mathfrak{U} / \operatorname{Rad} \mathfrak{A}$ is either: (i) an associative division algebra $\Delta$ with involution, (ii) a direct sum $\Delta \Delta^{\circ}$ of an associative division algebra with its opposite under the exchange involution, (iii) a split quaternion algebra $\Phi_{2}$ over its center $\Phi$ with standard involution.

Proof. $\quad \overline{\mathfrak{N}}=\mathfrak{N} / \operatorname{Rad} \mathfrak{A}$ is semisimple with involution, and $\overline{\mathfrak{J}} \subset \mathfrak{H}(\overline{\mathfrak{A}}, *)$ is still ample (but note that if $\mathfrak{J}=\mathfrak{H}(\mathfrak{U} *)$, it still may happen in characteristic 2 that $\overline{\mathfrak{I}}=\overline{\mathfrak{H}(\mathfrak{A}, *)}$ is not all of $\mathfrak{H}(\overline{\mathfrak{T}}, \bar{*})$, so we are forced to consider the case of a general ample subspace). Furthermore, $\overline{\mathfrak{J}}$ is semisimple as a Jordan subalgebra [7]. It therefore suffices to prove the result for $\overline{\mathfrak{A}}$ in place of $\mathfrak{A}$, i.e. we assume $\mathfrak{2}$ and $\mathfrak{J}$ are semisimple.

In this case $\mathfrak{A}$ is $*$-simple: if $\mathfrak{B}$ were a proper $*$-ideal, then for each $b$ in $\mathfrak{B}$ the elements $(1-b)\left(1-b^{*}\right)=1-z$ and $\left(1-b^{*}\right)(1-b)=1-w$ are invertible (since $z=b+b^{*}-b b^{*}$ and $w=b+b^{*}-b^{*} b$ lie in $\mathfrak{B} \cap \mathfrak{J}$, and cannot be invertible if $\mathfrak{B}$ is proper, so must be nilpotent); thus $1-b$ is invertible, each $b$ is quasi-invertible, and $\mathfrak{B} \subset \operatorname{Rad} \mathfrak{I}=0$. 
If the elements of $\mathfrak{J}$ are all invertible then by the Herstein-KleinfeldOsborn Theorem [3], $\mathfrak{A}$ is $\Delta, \Delta \oplus \Delta^{\circ}$, or $\Phi_{2}$. If the elements of $\mathfrak{I}$ are not all invertible then, by Theorem $1, x^{2}=Q(x) 1$ for all $x$ in $\mathfrak{I} \cong J(Q, 1)$, and we can go back to the situation where $z, y$ in $\mathfrak{J}$ satisfy $z^{2}=y^{2}=0, z \circ y=1$. Then $z y+y z=1, z=z y z, y=y z y$ so the elements $e_{11}=z y, e_{22}=y z, e_{12}=z$, $e_{21}=y$ are a family of matrix units. This gives us $\mathfrak{A} \cong \mathfrak{D}_{2}$ for $\mathfrak{D}=e_{11} \mathfrak{A} e_{11}$ under the correspondence $x=a+b e_{12}+e_{21} c+e_{21} d e_{12} \leftrightarrow\left(\begin{array}{ll}a & b \\ c & d\end{array}\right)$. Here $\mathfrak{I}$ contains $z \cong e_{12}$ and $y \cong e_{21}$. D inherits an involution $a \rightarrow \bar{a}=e_{12} a^{*} e_{21}$ (because $e_{12}^{*}=e_{12}, e_{21}^{*}=e_{21}, e_{11}^{*}=e_{22}$ if $z^{*}=z, y^{*}=y$ ), relative to which the involution $x \rightarrow x^{*}$ in $\mathfrak{A}$ corresponds to

$$
\left(\begin{array}{ll}
a & b \\
c & d
\end{array}\right) \rightarrow\left(\begin{array}{ll}
\bar{d} & \bar{b} \\
\bar{c} & \bar{a}
\end{array}\right)
$$

in $\mathfrak{D}_{2}$. Here $\mathfrak{H}\left(\mathfrak{D}_{2}, *\right)$ consists of the matrices $\left(\begin{array}{cc}a & h \\ k & a\end{array}\right)$ for $a \in \mathfrak{D}, h, k \in$ $\mathfrak{H}(\mathfrak{D},-)$.

The condition $x^{2}=Q(x) 1$ implies $x^{2} \circ y=0$ for any $x, y$ in $\mathfrak{J}$ or $x^{2} y=$ $y x^{2}$ (characteristic 2 !!). Observe that $\left(\begin{array}{ll}d & 0 \\ 0 & d\end{array}\right)$ belongs to $\mathfrak{I}$ since it is the trace of $\left(\begin{array}{ll}d & 0 \\ 0 & 0\end{array}\right)$ for any $d$ in $\mathfrak{D}$. Thus

$$
\left(\begin{array}{ll}
a & 0 \\
0 & \bar{a}
\end{array}\right)^{2}=\left(\begin{array}{cc}
a^{2} & 0 \\
0 & \bar{a}^{2}
\end{array}\right)
$$

commutes with $\left(\begin{array}{ll}b & 0 \\ 0 & b\end{array}\right)$ for any $a, b$ in $\mathfrak{D}$, and also with $z=\left(\begin{array}{ll}0 & 1 \\ 1 & 0\end{array}\right)$, so $a^{2} b=b a^{2}$ and $a^{2}=\bar{a}^{2}$, which shows that any $a^{2}$ lies in the subfield $\Phi=\mathfrak{S} \cap \Gamma$ of symmetric elements of the center $\Gamma$ of $\mathfrak{D}$. At the same time, any $\left(\begin{array}{cc}a \bar{a} & 0 \\ 0 & a \bar{a}\end{array}\right)$ belongs to $\mathfrak{J}$ as the norm of $\left(\begin{array}{ll}0 & a \\ a & 0\end{array}\right)$ so it commutes with all the $\left(\begin{array}{ll}b & 0 \\ 0 & b\end{array}\right)$ for $a, b$ in $\mathfrak{D}$, so $(a \bar{a}) b=b(a \bar{a})$ and all norms lie in the center of $\mathfrak{D}$. Therefore $\mathfrak{D}$ is a composition algebra over $\Phi$ with the additional property that $a^{2} \in \Phi$ for all $a$ in $\mathfrak{D}$; the only composition algebra with this property is $\mathfrak{D}=\Phi$, and our original algebra was a split quaternion algebra $\mathfrak{A}=\Phi_{2}$ with standard involution

$$
\left(\begin{array}{ll}
\alpha & \beta \\
\gamma & \delta
\end{array}\right) \rightarrow\left(\begin{array}{ll}
\delta & \beta \\
\gamma & \alpha
\end{array}\right)=\left(\begin{array}{cc}
\delta & -\beta \\
-\gamma & \alpha
\end{array}\right)
$$

(characteristic 2 !!).

Note. Professors I. N. Herstein and M. S. Montgomery have recently improved Osborn's Theorem in several directions [10]. One of their results is

THEOREM 3. If $\mathfrak{A}$ is an associative algebra with involution * such that each trace $x+x^{*}$ is either invertible or nilpotent, then $\mathfrak{U} / \operatorname{Rad} \mathfrak{U}$ is one of (1) a division ring $\Delta,(2)$ a direct sum $\Delta \oplus \Delta^{\circ}$ of a division ring with its opposite, 
(3) a split quaternion algebra $\Phi_{2}$ over its center, or (4) a commutative algebra of characteristic 2 with trivial involution.

The generalization consists in repacing the set of all symmetric elements by the set of traces. (As usual, this makes a difference only when $\frac{1}{2} \notin \Phi$.) The set $\mathfrak{I}(\mathfrak{A}, *)$ of traces is not necessarily an ample subspace of $\mathfrak{H}(\mathfrak{A}, *)$. We always have $x \mathfrak{I} x^{*} \subset \mathfrak{I}$ since $x t(y) x^{*}=x\left(y+y^{*}\right) x^{*}=t\left(x y x^{*}\right)$. However, we need not have $1 \in \mathfrak{I}$ (and consequently $\mathfrak{I}$ need not contain all norms $\left.x x^{*}\right)$. In particular, $\mathfrak{I}$ need not be closed under squaring and so cannot be considered as a Jordan algebra $\mathfrak{J}$. Thus our results cannot be applied directly to this situation.

However, there is a way to reduce the case of traces to the case of norms. The idea is that whenever 1 is a trace, $y+y^{*}=1$, then $\mathfrak{I}$ becomes an ample subspace (and contains all norms $x x^{*}=x t(y) x^{*}=t\left(x y x^{*}\right)$ ), so our result applies. If the unit of $\mathfrak{P}$ is not a trace, we pass to an isotope $\mathfrak{A}^{(u)}$ whose unit $u^{-1}$ is a trace; if the conclusions of Theorem 3 hold for $\mathfrak{A}^{(u)}$ they also hold for $\mathfrak{A}$.

To be more explicit, let us assume all traces $\mathfrak{I}(\mathfrak{H}, *)$ are invertible or nilpotent, where $\mathfrak{H}$ and $\mathfrak{H}(\mathfrak{H}, *)$ are semisimple. If all traces are zero, $x^{*}=-x$, then $\mathfrak{A}$ is commutative of characteristic 2 with trivial involution, and we have (4) of the theorem. If there are nonzero traces they cannot all be nilpotent, because of the general

LEMmA 4. If $z$ is an element of a Jordan algebra $\mathfrak{J}$ such that $z^{2}=0$ and $z \circ x$ is nilpotent, then $z$ is nilpotent in $\mathfrak{J}^{(x)}$. If this happens for all $x$ in $\mathfrak{J}$ then $z$ belongs to $\operatorname{Rad} \mathfrak{J}$.

Proof. If $z^{2}=0$ then $z^{3}$ is trivial $\left(U_{z^{3}}=0\right)$, so we may assume $z^{3}=0$ too (for example, by dividing out the lower radical [5]). Then $U_{z}^{(x)}=U_{z} U_{x}$ is nilpotent because $\left(U_{z} U_{x}\right) U_{z}=U_{x \odot z} U_{z}$ implies $\left(U_{z} U_{x}\right)^{n+1}=U_{z \circ x}^{n} U_{z} U_{x}$ (note $U_{z \circ x}=U_{z} U_{x}+U_{x} U_{z}+U_{z, x}^{2}-U_{z^{2}, x^{2}}$ where $U_{z} U_{z}=U_{z^{2}}=0$ and $U_{z, x} U_{z}=$ $U_{z^{2}, x} V_{z}-U_{z^{3}, x}=0$ if $z^{2}=z^{3}=0$ ). Consequently $z$ is nilpotent in the homotope $\mathfrak{J}^{(x)}$. If $z$ is quasi-invertible in all $\mathfrak{J}^{(x)}$ then it belongs to $\operatorname{Rad} \mathfrak{J}$ by [6].

The lemma applies to our $\mathfrak{J}=\mathfrak{H}(\mathfrak{H}, *)$ since if $z$ is a trace so are all $z x+$ $x z$ for $x$ in $\mathfrak{H}(\mathfrak{R}, *)$. From this we conclude that if a trace $z$ is nilpotent it squares to zero: the associative $*$-algebra $\mathfrak{B}=z \mathfrak{N} z$ has all its traces nilpotent (no $t(z a z)=z t(a) z$ can be invertible), so by the above is a radical algebra; then all $(z a) z$ are quasi-invertible, so all $z^{2} a$ are too, and $z^{2}$ lies in $\operatorname{Rad} \mathfrak{R}=0$.

Therefore some trace $x+x^{*}=u^{-1}$ is invertible. The isotope $\mathfrak{A}^{(u)}$ (with multiplication $x \cdot{ }_{u} y=x u y$ ) has unit $1^{(u)}=u^{-1}$ a trace. Since invertible elements of $\mathfrak{O}$ are invertible in $\mathfrak{I}^{(u)}$, if nilpotent traces in $\mathfrak{P}$ stay nilpotent in $\mathfrak{I}^{(u)}$ then Theorem 2 will apply to $\mathfrak{P I}^{(u)}$ to give (1), (2), or (3). 
On the other hand, if some nilpotent trace $z$ does not stay nilpotent in $\mathfrak{A}^{(u)}$ we claim $1^{\prime}$ is already a trace in $\mathfrak{A}$, so Theorem 2 applies to $\mathfrak{A}$ itself. Indeed, since $z^{2}=0$ but $z$ is not nilpotent in $\mathfrak{A}^{(u)}$, by Lemma 4 the trace $z \circ u$ must not be nilpotent in $\mathfrak{A}$, consequently $z u+u z=v$ is invertible. Now $u z u$ is a trace which is not invertible, so it squares to zero, and $z u^{2} z=$ $u^{-1}(u z u)^{2} u^{-1}=0$. Note $z v=z u z=v z$ since $z^{2}=0$. Then $v^{2}=(z u+u z)^{2}=$ $z u z u+u z u z=v z u+u z v$, so $1=v^{-1}\left(v^{2}\right) v^{-1}=z u v^{-1}+v^{-1} u z$ is a trace $(u, v, z$ being symmetric).

An even better trick is due to Professor Tamagawa. Instead of keeping the involution the same but changing the algebra to $\mathfrak{H}^{(u)}$, he keeps the algebra the same but changes the involution to $x^{*(u)}=u x^{*} u^{-1}$. Here $T(\mathfrak{A}, *(u))=$ $u T(\mathfrak{A}, *)$ since $x+x^{*(u)}=x+u x^{*} u^{-1}=u\left[\left(u^{-1} x\right)+\left(u^{-1} x\right)^{*}\right]$, so if $u^{-1} \in$ $T(\mathfrak{A}, *)$ then $1 \in T(\mathfrak{H}, *(u))$. The problem of showing the elements of $u T(\mathfrak{A}, *)$ are invertible or nilpotent in $\mathfrak{A}$ is equivalent to showing the elements of $T(\mathfrak{A}, *)$ are invertible or nilpotent in $\mathfrak{P}^{(u)}$.

We remark that the trick of passing to an isotope is not available where traces are merely regular or nilpotent.

\section{REFERENCES}

1. N. Jacobson, $A$ theorem on the structure of Jordan algebras, Proc. Nat. Acad. Sci. U.S.A. 42 (1956), 140-147. MR 17, 822.

2. -_- Structure and representations of Jordan algebras, Amer. Math. Soc. Colloq. Publ., vol. 39, Amer. Math. Soc., Providence, R.I., 1968. MR 40 \#4330.

3. - Lectures on quadratic Jordan algebras, Tata Institute Lecture Notes, Bombay, 1970.

4. N. Jacobson and K. McCrimmon, Quadratic Jordan algebras of quadratic forms with base points, Indian Math. J. (to appear).

5. K. McCrimmon, The radical of a Jordan algebra, Proc. Nat. Acad. Sci. U.S.A. 62 (1969), 671-678. MR 42 \#3137.

6. -_-, A characterization of the radical of a Jordan algebra, J. Algebra 18 (1971), $103-111$.

7. - On Herstein's theorems relating Jordan and associative algebras, J. Algebra 13 (1969), 382-392. MR 40 \#2721.

8. D. L. Morgan, Jordan algebras with minimum condition, Trans. Amer. Math. Soc. 155 (1971), 161-173. MR 43 \#2037.

9. J. M. Osborn, Jordan and associative rings with nilpotent and invertible elements, J. Algebra 15 (1970), 301-308. MR 41 \#6925.

10. I. N. Herstein and M. S. Montgomery, Invertible and regular elements in rings with involution (to appear).

Department of Mathematics, University of Virginia, Charlottesville, VirGINIA 22904 